Draft VERSion MARCh 21, 2018

Preprint typeset using LATEX style AASTeX6 v. 1.0

\title{
THE SLUGGS SURVEY: A CATALOG OF OVER 4000 GLOBULAR CLUSTER RADIAL VELOCITIES IN 27 NEARBY EARLY-TYPE GALAXIES
}

\author{
Duncan A. Forbes ${ }^{1}$, Adebusola Alabi ${ }^{1}$, Jean P. Brodie ${ }^{2}$, Aaron J. Romanowsky ${ }^{2,3}$, Jay Strader ${ }^{4}$, Caroline \\ Foster $^{5}$, Christopher Usher ${ }^{7}$, Lee Spitler ${ }^{5,6}$, Sabine Bellstedt $^{1}$, Nicola Pastorello ${ }^{1,8}$, Alexa Villaume ${ }^{2}$, Asher $^{2}$ \\ WASSERman ${ }^{2}$, Vincenzo Pota ${ }^{9}$ \\ ${ }^{1}$ Centre for Astrophysics \& Supercomputing, Swinburne University, Hawthorn, VIC 3122, Australia; dforbes@swin.edu.au \\ ${ }^{2}$ University of California Observatories, 1156 High Street, Santa Cruz, CA 95064, USA
}

${ }^{3}$ Department of Physics and Astronomy, San José State University, One Washington Square, San Jose, CA 95192, USA

${ }^{4}$ Department of Physics and Astronomy, Michigan State University, East Lansing, Michigan 48824, USA

${ }^{5}$ Australian Astronomical Observatory, PO Box 915, North Ryde, NSW 1670, Australia

${ }^{6}$ Macquarie Research Centre for Astronomy, Astrophysics \& Astrophotonics, Macquarie University, Sydney, NSW 2109, Australia

${ }^{7}$ Astrophysics Research Institute, Liverpool John Moores University, 146 Brownlow Hill, Liverpool L3 5RF, UK

${ }^{8}$ Deakin Software and Technology Innovation Laboratory, Deakin University, Burwood, VIC 3125, Australia

and

${ }^{9}$ INAF - Osservatorio Astronomico di Capodimonte, Salita Moiariello, 16, 80131 Napoli, Italy

\begin{abstract}
Here we present positions and radial velocities for over 4000 globular clusters (GCs) in 27 nearby early-type galaxies from the SLUGGS survey. The SLUGGS survey is designed to be representative of elliptical and lenticular galaxies in the stellar mass range $10<\log \mathrm{M}_{*} / \mathrm{M}_{\odot}<11.7$. The data have been obtained over many years, mostly using the very stable multi-object spectrograph DEIMOS on the Keck II 10m telescope. Radial velocities are measured using the calcium triplet lines with a velocity accuracy of $\pm 10-15 \mathrm{~km} / \mathrm{s}$. We use phase space diagrams (i.e. velocity-position diagrams) to identify contaminants such as foreground stars and background galaxies, and to show that the contribution of GCs from neighboring galaxies is generally insignificant. Likely ultra-compact dwarfs are tabulated separately. We find that the mean velocity of the GC system is close to that of the host galaxy systemic velocity, indicating that the GC system is in overall dynamical equilibrium within the galaxy potential. We also find that the GC system velocity dispersion scales with host galaxy stellar mass in a similar manner to the Faber-Jackson relation for the stellar velocity dispersion. Publication of these GC radial velocity catalogs should enable further studies in many areas, such as GC system substructure, kinematics, and host galaxy mass measurements.
\end{abstract}


Keywords: catalogs — surveys - galaxies: star clusters

\section{INTRODUCTION}

Radial velocities for globular clusters (GCs) beyond the Local Group were first published in the 1980s (Hesser et al. 1986; Huchra \& Brodie 1987; Mould et al. 1987). Although these studies typically had individual GC velocity uncertainties of $\geq 50 \mathrm{~km} \mathrm{~s}^{-1}$, they quickly showed the benefit of spectroscopically confirming GC candidates. For example, several of the brightest GC candidates around M87 from the imaging study of Strom et al. (1981) were shown to be background galaxies by Huchra \& Brodie (1984).

As well as confirming that candidates from imaging are indeed bona fide GCs, radial velocities were employed to probe GC kinematics relative to the host galaxy (Hesser et al. 1986), investigate the velocity dispersion profile in the galaxy halo (Mould et al. 1987) and derive the enclosed mass to large radii (Huchra \& Brodie 1987).

Globular cluster radial velocity studies have tended to focus on a small number of nearby massive early-type galaxies with rich GC systems, e.g. NGC 1316 (Richtler et al. 2014), NGC 1399 (Schuberth et al. 2010), NGC 3311 (Richtler et al. 2011; Misgeld et al. 2011), NGC 5128 (Beasley et al. 2008; Woodley et al. 2010), NGC 4472 (M49, Zepf et al. 2000; Cote et al. 2003), NGC 4486 (M87, Cote et al. 2001; Strader et al. 2011), NGC 4594 (M104, Bridges et al. 2007; Dowell et al. 2014), NGC 4636 (Schuberth et al. 2012). The number of GCs studied in a given system and the typical velocity uncertainty have improved since the earlier studies of the 1980s and 1990s. However, very few lower mass early-type galaxies had been studied by the mid 2000s.

The dual aims of the SLUGGS survey (Brodie et al. 2014) are to collect high quality GC and galaxy starlight spectra for a representative sample of early-type galaxies over a wide range of stellar mass (i.e. $10<\log \mathrm{M}_{*} / \mathrm{M}_{\odot}<$ 11.7). The galaxy starlight spectra are used to probe the kinematics and metallicity of the host galaxy (see Brodie et al. 2014 for details) and have been reported elsewhere in the literature (see www.sluggs.swin.edu.au). Over the last decade we have obtained over $4000 \mathrm{GC}$ radial velocities associated with the 25 main galaxies, and 'bonus' galaxies, of the survey. Results have been published on a continuous basis over the years. This includes GC kinematics of individual galaxies (NGC 1407, Romanowsky et al. 2009; NGC 4494, Foster et al. 2011; NGC 4473, Alabi et al. 2015; NGC 4649, Pota et al. 2015), interacting galaxies (NGC 3607 and NGC 3608, Kartha et al. 2016), and a sample of a dozen galaxies (Pota et al. 2013). We have also used GC kinematics to derive mass models of the host galaxy, thereby exploring its dark matter content (Napolitano et al. 2014; Pota et al. 2015; Alabi et al. 2016).

In the next section we summarise the SLUGGS earlytype galaxy sample and the observational setup used. We then discuss the removal of potential contaminants and present the final GC radial velocity catalogs.

\section{THE HOST GALAXY SAMPLE AND OBSERVATIONS}

Our sample consists of GC systems associated with 25 early-type galaxies from the SLUGGS survey plus two of the three bonus galaxies (NGC 3607 and NGC 5866) that were observed with the same setup. Table 1 lists the 27 galaxies and some relevant properties, such as their distance, stellar mass, effective radius, morphology, environment, systemic velocity, stellar velocity dispersion within $1 \mathrm{kpc}$, and position (J2000 coordinates). Most of these properties are taken from Brodie et al. (2014), which also lists other properties of the galaxies.

We have obtained wide-field multi-filter imaging of the SLUGGS galaxies using the Subaru telescope under $\leq 1$ arcsec seeing conditions. This is supplemented by HST and CFHT imaging. Publications presenting the imaging analysis of SLUGGS galaxies include NGC 1407 (Romanowsky et al. 2009), NGC 4365 (Blom et al. 2012), NGC 4278 (Usher et al. 2013), NGC 720, 1023 and 2768 (Kartha et al. 2014), NGC 1023 (Forbes et al. 2014), NGC 3115 (Jennings et al. 2014), and NGC 3607 and 3608 (Kartha et al. 2016). We plan to publish an imaging analysis of the GC systems of the remaining SLUGGS galaxies in due course.

Spectroscopic observations of GC candidates were obtained over the last decade using the DEIMOS spectrograph (Faber et al. 2003) on the Keck II 10m telescope. The DEIMOS instrument is used in multi-slit mode with each slit mask covering an area of $\sim 16 \times 5 \operatorname{arcmin}^{2}$. With a flexure compensation system, DEIMOS is a very stable instrument and ideal for obtaining red spectra of objects over a wide field-of-view. For the SLUGGS survey, we use the 1200 lines per mm grating, the OG550 filter, slit width of 1 arcsec and a central wavelength of $7800 \AA$. This gives $50-100$ spectra per mask around the calcium triplet $(\mathrm{CaT})$ feature covering a wavelength range of $\sim 6500 \AA$ to $9000 \AA$. Each mask targets either $\mathrm{GC}$ candidates or locations near the galaxy center in order to obtain spectra of the underlying galaxy starlight. Globular clusters are selected to cover the full range of expected colors but have a bias towards the brighter objects in a given GC system (in order to maximise the 
signal-to-noise). Our setup has a spectral resolution of $\sim \quad$ ing conditions of typically $\leq 1$ arcsec. $1.5 \AA$ (FWHM). Observations were obtained under see-

Table 1. SLUGGS Galaxy Properties

\begin{tabular}{|c|c|c|c|c|c|c|c|c|c|}
\hline $\begin{array}{l}\text { Galaxy } \\
\text { (NGC) }\end{array}$ & $\begin{array}{l}\text { Dist. } \\
(\mathrm{Mpc})\end{array}$ & $\begin{array}{c}\log \mathrm{M}_{*} \\
\left(\mathrm{M}_{\odot}\right)\end{array}$ & $\begin{array}{c}\mathrm{R}_{e} \\
(\operatorname{arcsec})\end{array}$ & Type & Env. & $\begin{array}{c}\mathrm{V}_{\text {sys }} \\
(\mathrm{km} / \mathrm{s})\end{array}$ & $\begin{array}{c}\sigma_{k p c} \\
(\mathrm{~km} / \mathrm{s})\end{array}$ & $\begin{array}{l}\text { (R.A.) } \\
\text { (deg.) }\end{array}$ & $\begin{array}{l}\text { (Dec.) } \\
\text { (deg.) }\end{array}$ \\
\hline 720 & 26.9 & 11.27 & 29.1 & E5 & $\mathrm{F}$ & 1745 & 227 & 28.252077 & -13.738653 \\
\hline 821 & 23.4 & 11.00 & 43.2 & E6 & $\mathrm{F}$ & 1718 & 193 & 32.088083 & 10.994917 \\
\hline 1023 & 11.1 & 10.99 & 48.0 & S0 & G & 602 & 183 & 40.100042 & 39.063285 \\
\hline 1400 & 26.8 & 11.08 & 25.6 & E1/S0 & G & 558 & 236 & 54.878483 & -18.688070 \\
\hline 1407 & 26.8 & 11.60 & 93.4 & E0 & G & 1779 & 252 & 55.049417 & -18.580111 \\
\hline 2768 & 21.8 & 11.21 & 60.3 & E6/S0 & G & 1353 & 206 & 137.906250 & 60.037222 \\
\hline 2974 & 20.9 & 10.93 & 30.2 & $\mathrm{E} 4 / \mathrm{S} 0$ & $\mathrm{~F}$ & 1887 & 231 & 145.638667 & -3.699139 \\
\hline 3115 & 9.4 & 10.93 & 36.5 & S0 & $\mathrm{F}$ & 663 & 248 & 151.308250 & -7.718583 \\
\hline 3377 & 10.9 & 10.50 & 45.4 & E5-6 & G & 690 & 135 & 161.926380 & 13.985916 \\
\hline 3607 & 22.2 & 11.39 & 48.2 & So & G & 942 & 229 & 169.227665 & 18.051756 \\
\hline 3608 & 22.3 & 11.03 & 42.9 & E1-2 & G & 1226 & 179 & 169.245632 & 18.148684 \\
\hline 4111 & 14.6 & 10.52 & 10.1 & So & G & 792 & 161 & 181.763052 & 43.065720 \\
\hline 4278 & 15.6 & 10.95 & 28.3 & E1-2 & G & 620 & 228 & 185.028434 & 29.280756 \\
\hline 4365 & 23.1 & 11.51 & 77.8 & E3 & G & 1243 & 253 & 186.117852 & 7.3176728 \\
\hline 4374 & 18.5 & 11.51 & 139.0 & E1 & $\mathrm{C}$ & 1017 & 284 & 186.265597 & 12.886983 \\
\hline 4459 & 16.0 & 10.98 & 48.3 & S0 & $\mathrm{C}$ & 1192 & 170 & 187.250037 & 13.978373 \\
\hline 4473 & 15.2 & 10.96 & 30.2 & E5 & $\mathrm{C}$ & 2260 & 189 & 187.453628 & 13.429359 \\
\hline 4474 & 15.5 & 10.23 & 17.0 & S0 & $\mathrm{C}$ & 1611 & 88 & 187.473113 & 14.068589 \\
\hline 4486 & 16.7 & 11.62 & 86.6 & $\mathrm{E} 0 / \mathrm{cD}$ & $\mathrm{C}$ & 1284 & 307 & 187.705930 & 12.391123 \\
\hline 4494 & 16.6 & 11.02 & 52.5 & E1-2 & G & 1342 & 157 & 187.850433 & 25.775252 \\
\hline 4526 & 16.4 & 11.26 & 32.4 & S0 & $\mathrm{C}$ & 617 & 233 & 188.512856 & 7.6995240 \\
\hline 4564 & 15.9 & 10.58 & 14.8 & E6 & $\mathrm{C}$ & 1155 & 153 & 189.112428 & 11.439283 \\
\hline 4649 & 16.5 & 11.60 & 79.2 & $\mathrm{E} 2 / \mathrm{S} 0$ & $\mathrm{C}$ & 1110 & 308 & 190.916564 & 11.552706 \\
\hline 4697 & 12.5 & 11.15 & 95.8 & E6 & G & 1252 & 180 & 192.149491 & -5.8007419 \\
\hline 5846 & 24.2 & 11.46 & 89.8 & E0-1/S0 & G & 1712 & 231 & 226.622017 & 1.6056250 \\
\hline 5866 & 14.9 & 10.83 & 23.4 & S0 & G & 755 & 163 & 226.622912 & 55.763213 \\
\hline 7457 & 12.9 & 10.13 & 34.1 & So & $\mathrm{F}$ & 844 & 74 & 345.249726 & 30.144941 \\
\hline
\end{tabular}

Note-Distance, morphology, environment, galaxy systemic velocity and velocity dispersion within $1 \mathrm{kpc}$ are taken from Brodie et al. (2014). Stellar masses and effective radii are from Forbes et al. (2016). Note that $\mathrm{V}_{\text {sys }}$ for NGC 4474 was reported incorrectly in Brodie et al. and has been corrected here. The position of each galaxy centre is taken from the NASA Extragalactic Database.

The spectra are reduced using the spec2d data reduction pipeline (Cooper et al. 2012) which produces skysubtracted, wavelength calibrated spectra. We use FXCOR (Tonry \& Davies 1979) within IRAF, along with 13 stellar template spectra (observed with DEIMOS in the same setup but in long slit mode), to determine the radial velocity of each object. Velocity errors are the quadrature combination of the FXCOR error and the standard deviation from the 13 stellar templates (which cover a range of metallicity and spectral type), which give a minimum measurement uncertainty of $\pm 3 \mathrm{~km} \mathrm{~s}^{-1}$. We visually check each spectrum and require that at least two of the three CaT lines (8498 $, 8542 \AA, 8662 \AA)$ and $\mathrm{H} \alpha$ (if included in the redshifted spectrum) are 
present. A small percentage of the spectra are 'marginal' in the sense that we can not be sure about the identification of the lines (e.g. due to low $\mathrm{S} / \mathrm{N}$ or poor sky subtraction). In these cases we take a conservative approach and do not include them in our confirmed GC catalogs (nor those of confirmed contaminants). Radial velocities are corrected to heliocentric velocities. Our tests of repeatability (i.e. from observing the same objects on different nights) indicates a systematic rms velocity uncertainty of $\pm 10-15 \mathrm{~km} / \mathrm{s}$ (Pota et al. 2013, 2015).

\section{BACKGROUND GALAXIES AND FOREGROUND STARS}

Our initial GC candidate selection is largely based on ground-based imaging which will include some contaminants, i.e. both compact background galaxies and foreground stars. By examining phase space diagrams, i.e. the radial velocity vs galactocentric radius of the $\mathrm{GC}$ candidates (see the Appendix for such diagrams of each galaxy's GC system), it is fairly straight forward to identify and remove background galaxies on the basis of their high velocities i.e. $\mathrm{V}>3000 \mathrm{~km} \mathrm{~s}^{-1}$ (from either absorption or emission lines).

For most GC systems, the GCs are also well separated in velocity from the most extreme Milky Way stars, which generally have velocities within $\pm 300 \mathrm{~km} \mathrm{~s}^{-1}$ (although some rare examples of very high velocity halo stars do exist; Brown et al. 2010). For the half dozen GC systems that may overlap in velocity with Milky Way stars, one can assume that the GC velocities are distributed symmetrically about the galaxy's systemic velocity and use those GCs with higher than systemic velocity to define the distribution (see for example Usher et al. 2013). Extending that velocity distribution to velocities less than the galaxy's systemic velocity, gives an indication of likely foreground star contaminants. An additional clue comes from the object's galactocentric radius since the velocity dispersion of a $\mathrm{GC}$ system tends to decrease with radius; very few GC system phase diagrams have objects with $\mathrm{V}<300 \mathrm{~km} \mathrm{~s}^{-1}$ at large radii.

Our final GC catalogs are thus our best effort at removing foreground stars and background galaxies but a small number of such contaminants may still be present. We do not attempt to remove any GCs associated with substructures within a GC system that may have been acquired from a merger/accretion event (see Alabi et al. 2016 for a discussion of this issue). The exception to this is NGC $4365\left(\mathrm{~V}_{\text {sys }}=\right.$ $1243 \mathrm{~km} \mathrm{~s}^{-1}$ ) for which GCs deemed to be associated with the interacting galaxy NGC $4342\left(\mathrm{~V}_{\text {sys }}=761\right.$ $\mathrm{km} \mathrm{s}^{-1}$ ) have been removed (see Blom et al. 2014 for details). Table 2 lists foreground star and background galaxy contaminants (we no not quote actual recession velocities for background galaxies as we only applied absorption line templates) identified for each SLUGGS galaxy (excluding NGC 4486, 4494 and 4649).

Table 2. Contaminants

\begin{tabular}{lcccc}
\hline \hline ID & $\begin{array}{c}\text { R.A. } \\
(\text { deg. })\end{array}$ & $\begin{array}{c}\text { Dec. } \\
\text { (deg. })\end{array}$ & $\begin{array}{c}\mathrm{V} \\
(\mathrm{km} / \mathrm{s})\end{array}$ & $\begin{array}{c}\mathrm{V}_{e} \\
(\mathrm{~km} / \mathrm{s})\end{array}$ \\
& & & & \\
\hline NGC720_star1 & 28.166625 & -13.666556 & 158 & 7 \\
NGC720_star2 & 28.221083 & -13.783722 & 6 & 5 \\
$\ldots$ & $\ldots$ & $\ldots$ & $\ldots$ & $\ldots$ \\
NGC720_gal1 & 28.231667 & -13.773028 & 99 & 99 \\
& & & & \\
\hline
\end{tabular}

Note-ID, Right Ascension and Declination (J2000), heliocentric radial velocity and velocity uncertainty. Velocities and velocity uncertainties of 99 denote no measured value. The full table is published online.

\section{NEIGHBORING GALAXIES}

A neighboring galaxy may also possess its own GC system which, if close in projection on the sky and in radial velocity, could be confused with that of the primary SLUGGS galaxy. For most of the SLUGGS galaxies, there is no nearby neighbor of substantial size and hence rich GC system. The main exception is the Leo II galaxy group. Here we have used HST and Subaru imaging along with the spectroscopically-confirmed GCs to remove any GCs likely associated with the dwarf galaxy NGC 3605 and assign the bulk of GCs to either NGC 3607 or NGC 3608 (Kartha et al. 2016). GCs identified as being associated with NGC 4459 may in principle belong to the very rich GC system of nearby NGC 4486 (M87). For NGC 4459, the bulk of its GCs lie within $\sim 2$ galaxy effective radii but some half a dozen objects lie at large radii and may actually belong to M87. For NGC 4278 we include here, the 3 GCs that may be associated with NGC 4283 as identified by Usher et al. (2013). For NGC 1407 and NGC 1400, the galaxies are separated by over $1000 \mathrm{~km} \mathrm{~s}^{-1}$ in velocity and 10 arcminutes on the sky, so it is straight forward to assign their relative GC systems. Otherwise the neighboring galaxies tend to be low mass galaxies and/or located at large projected galactocentric radii. Table 3 lists potential neighbor galaxies that are projected within 12 arcmins, differ by less than $1000 \mathrm{~km} / \mathrm{s}$ in systemic velocity and are less than 4 magnitudes different from the primary SLUGGS galaxy. From our phase-space diagrams (see the Appendix) the contribution from neighboring galaxies' GC systems appear to be small and we have not attempted 
to remove any such GCs from the SLUGGS galaxy GC system.

Table 3. Neighbor galaxies

\begin{tabular}{|c|c|c|c|}
\hline $\begin{array}{l}\text { Galaxy } \\
(\mathrm{NGC})\end{array}$ & Neighbor galaxy & $\begin{array}{l}\Delta \mathrm{V}_{\text {sys }} \\
(\mathrm{km} / \mathrm{s})\end{array}$ & $\begin{array}{c}\Delta \mathrm{R} \\
(\operatorname{arcmin})\end{array}$ \\
\hline 3377 & NGC 3377A & 117 & 7.0 \\
\hline 3607 & NGC 3605 & 281 & 2.8 \\
\hline 3607 & NGC 3608 & -284 & 5.9 \\
\hline 3608 & NGC 3607 & 284 & 5.9 \\
\hline 3608 & NGC 3605 & 565 & 8.4 \\
\hline 4111 & NGC 4117 & -142 & 8.6 \\
\hline 4111 & UGC 07094 & 13 & 11.6 \\
\hline 4278 & NGC 4283 & -436 & 3.5 \\
\hline 4278 & NGC 4286 & -24 & 8.6 \\
\hline 4365 & NGC 4366 & -33 & 5.1 \\
\hline 4365 & NGC 4370 & 461 & 10.1 \\
\hline 4374 & NGC 4387 & 452 & 10.3 \\
\hline 4459 & NGC 4468 & 283 & 8.6 \\
\hline 4473 & NGC 4479 & 1384 & 11.4 \\
\hline 4474 & NGC 4468 & 702 & 5.6 \\
\hline 4486 & NGC 4478 & -65 & 8.7 \\
\hline 4649 & NGC 4647 & -299 & 2.6 \\
\hline 5846 & NGC 5846A & -489 & 0.6 \\
\hline 5846 & NGC 5845 & 240 & 7.3 \\
\hline 5846 & NGC 5850 & -844 & 10.3 \\
\hline 7457 & UGC 12311 & -76 & 7.8 \\
\hline
\end{tabular}

Note-Neighbor galaxies that lie within 12 arcmins on the sky, $<1000 \mathrm{~km} / \mathrm{s}$ in systemic velocity difference and $<4$ magnitudes difference, systemic velocity of SLUGGS galaxy minus that of the neighbor and projected distance on the sky.

\section{ULTRA-COMPACT DWARFS}

As well as removing background galaxies and foreground stars from our GC object lists, we have attempted to remove an additional source of 'contamination' by Ultra-Compact Dwarfs (UCDs). UCDs appear very similar to GCs in ground-based imaging, and lack a standard definition. Working definitions have included half light sizes greater than $10 \mathrm{pc}$ and/or luminosities brighter than $\mathrm{M}_{V} \sim-11$ (i.e. on the order of $\omega$ Cen in our Galaxy). In order to measure sizes for objects around SLUGGS galaxies (which have typical distances of $20 \mathrm{Mpc}$ ) HST imaging is generally required, and not always available for our GC sample. Here we have taken a conservative approach of excluding the small number of GC-like objects with an equivalent luminosity of $\mathrm{M}_{i} \leq$ -12 (this roughly corresponds to $\mathrm{M}_{V}<-11$ and masses greater than two million solar masses); thus our GC object lists may still include a small number of low luminosity UCDs with sizes greater than $10 \mathrm{pc}$ (see Forbes et al. 2013). We tabulate the objects we identify as UCDs in Table 4 for the galaxies NGC 821, 1023, 1407, 2768, 4365, 4494 and 4649. We note that Table 4 includes the 3 objects identified as UCDs around NGC 4494 by Foster et al. (2011) even though they have luminosities of $\mathrm{M}_{i} \sim-11.8$, which is slightly fainter than our limit. For a discussion of UCDs around NGC 4486 (M87) we refer the interested reader to Strader et al. (2011). We adopt a naming convention of NGCXXXX_UCDXX, i.e. the galaxy NGC name and a sequence of identified ultracompact dwarfs.

Table 4. Ultra-Compact Dwarf Radial Velocities

\begin{tabular}{lcclll}
\hline \hline UCD ID & R.A. & Dec. & V & $\mathrm{V}_{e}$ & $\mathrm{Rad}$ \\
& & & & & \\
\hline NGC821_UCD1 & 32.086091 & 10.990721 & 1705 & 6 & 0.28 \\
\hline NGC1023_UCD1 & 40.144680 & 39.090030 & 619 & 4 & 2.63 \\
NGC1023_UCD2 & 40.115950 & 39.078000 & 338 & 3 & 1.15 \\
\hline NGC1407_UCD1 & 55.007179 & -18.630067 & 2110 & 5 & 3.84 \\
NGC1407_UCD2 & 55.067500 & -18.481872 & 2164 & 5 & 5.98 \\
NGC1407_UCD3 & 55.065921 & -18.541622 & 1665 & 5 & 2.49 \\
NGC1407_UCD4 & 55.058625 & -18.641786 & 1482 & 5 & 3.74 \\
NGC1407_UCD5 & 55.089904 & -18.725344 & 1712 & 5 & 9.01 \\
NGC1407_UCD6 & 54.861854 & -18.688042 & 1995 & 6 & 12.5 \\
NGC1407_UCD7 & 55.041750 & -18.568922 & 1954 & 5 & 0.80 \\
NGC1407_UCD8 & 54.963000 & -18.485567 & 1621 & 35 & 7.51 \\
NGC1407_UCD9 & 55.096717 & -18.505539 & 1973 & 3 & 5.22 \\
NGC1407_UCD10 & 55.017663 & -18.562511 & 2509 & 4 & 2.09 \\
NGC1407_UCD11 & 55.039700 & -18.560778 & 1187 & 4 & 1.28 \\
\hline NGC2768_UCD1 & 137.903214 & 60.071148 & 1194 & 5 & 2.04 \\
\hline NGC4365_UCD1 & 186.096020 & 7.317350 & 1518 & 5 & 1.30 \\
NGC4365_UCD2 & 186.062140 & 7.320480 & 800 & 5 & 3.32 \\
NGC4365_UCD3 & 186.082990 & 7.300690 & 1446 & 5 & 2.31 \\
NGC4365_UCD4 & 186.110750 & 7.319560 & 979 & 5 & 0.44 \\
NGC4365_UCD5 & 186.148890 & 7.306630 & 1586 & 5 & 1.96 \\
NGC4365_UCD6 & 186.086620 & 7.311630 & 898 & 5 & 1.89 \\
NGC4365_UCD7 & 186.120030 & 7.366040 & 929 & 5 & 2.90 \\
\hline NGC4494_UCD1 & 187.856312 & 25.772158 & 1281 & 5 & 0.37 \\
NGC4494_UCD2 & 187.852679 & 25.804469 & 1341 & 5 & 1.77 \\
\hline & & & & & \\
\hline
\end{tabular}

Table 4 continued 
Table 4 (continued)

\begin{tabular}{cccllll}
\hline \hline \multirow{2}{*}{ UCD ID } & R.A. & Dec. & V & $\mathrm{V}_{e}$ & $\mathrm{Rad}$ \\
& & & & & \\
\hline NGC4649_UCD1 & 190.950662 & 11.534806 & 826 & 3 & 2.27 \\
NGC4649_UCD2 & 190.938146 & 11.589529 & 1275 & 4 & 2.55 \\
NGC4649_UCD3 & 190.912098 & 11.576443 & 796 & 25 & 1.45 \\
NGC4649_UCD4 & 190.700026 & 11.920495 & 1221 & 28 & 25.5 \\
NGC4649_UCD5 & 190.913204 & 11.549560 & 1526 & 8 & 0.27 \\
NGC4649_UCD6 & 191.042458 & 11.578678 & 1450 & 28 & 7.56 \\
NGC4649_UCD7 & 190.735808 & 11.619961 & 1227 & 22 & 11.4 \\
NGC4649_UCD8 & 190.788416 & 11.648972 & 1042 & 16 & 9.49 \\
& & & & & & \\
\hline
\end{tabular}

Note-Ultra-compact dwarf ID, Right Ascension and Declination (J2000), heliocentric radial velocity $(\mathrm{km} / \mathrm{s})$, velocity uncertainty $(\mathrm{km} / \mathrm{s})$ and galactocentric radius (arcmin).

\section{GLOBULAR CLUSTER RADIAL VELOCITY CATALOGS}

In Table 5 we present our GC radial velocity catalogs. Each catalog lists the globular cluster ID, its position, heliocentric radial velocity, velocity uncertainty and galactocentric radius (in arcminutes) for each SLUGGS galaxy. The position of each galaxy centre is given in Table 1. For object IDs we use a naming convention of NGCXXXX_SXXX, i.e. the galaxy NGC name and a sequence of SLUGGS velocity-confirmed globular clusters. We do not include any GCs that we determined to have marginal (i.e. non secure) measurements of their velocity. The catalog for NGC 3115 includes GCs observed by Arnold et al. (2011) using Keck/LRIS and Magellan/IMACS as well as Keck/DEIMOS. For NGC 4649, the catalog includes GCs observed using Gemini/GMOS, MMT/Hectospec as well as Keck/DEIMOS as complied by Pota et al. (2015). Our catalog for NGC 4486 includes GCs observed by the MMT/Hectospec, particularly at large galactocentric radii, as well as Keck /DEIMOS. See Strader et al. (2011) for details. Our Keck/DEIMOS observations of NGC 4365 were extended to include GCs around NGC 4342 which is separated by $\sim 20$ arcmin and $\sim 500 \mathrm{~km} / \mathrm{s}$ in velocity (Blom et al. 2014). Here we only include GCs associated with NGC 4365 and refer the reader to Blom et al. (2014) for the GCs associated with NGC 4342. When a GC has been observed multiple times, we list the average velocity value and average uncertainty (combining errors in quadrature). These new, updated catalogs presented in Table 5 supersede previous SLUGGS GC radial velocity catalogs (e.g. Usher et al. 2012; Pota et al. 2013).
Table 5. Globular Cluster Radial Velocities

\begin{tabular}{crrrrr}
\hline \hline NGC 720 & & & & & \\
\hline GC ID & R.A. & Dec. & V & $\mathrm{V}_{e}$ & Rad \\
& & & & & \\
\hline NGC720_S1 & 28.165375 & -13.732361 & 1794 & 11 & 5.07 \\
NGC720_S2 & 28.217625 & -13.731111 & 1805 & 10 & 2.06 \\
NGC720_S3 & 28.165917 & -13.715389 & 1772 & 11 & 5.21 \\
$\ldots$ & $\ldots$ & $\ldots$ & $\ldots$ & $\ldots$ & $\ldots$ \\
\hline
\end{tabular}

Note-Globular cluster ID, Right Ascension and Declination (J2000), heliocentric radial velocity $(\mathrm{km} / \mathrm{s})$, velocity uncertainty $(\mathrm{km} / \mathrm{s})$, galactocentric radius (arcmin). The full table is published online.

In Table 6 we summarise our final GC radial velocity catalogs. We list the number of unique DEIMOS masks and the total integration time. Note these masks were usually of dual purpose, i.e. as well as GCs, we obtained spectra of the underlying galaxy starlight to probe host galaxy kinematics (Arnold et al. 2014; Foster et al. 2016) and metallicity (Pastorello et al. 2014). If the emphasis of a given mask was on obtaining starlight then the GC return rate may be lower than if we had dedicated the mask to GCs. Table 6 also lists the number of unique confirmed GCs - this excludes those objects determined to be marginal GCs, background galaxies, foreground stars and UCDs. For each GC system we calculate the error-weighted mean heliocentric velocity along with its uncertainty, and the velocity dispersion (the standard deviation of the distribution).

Table 6. GC system catalog properties

\begin{tabular}{cccrcc}
\hline \hline $\begin{array}{c}\text { Galaxy } \\
\text { (NGC) }\end{array}$ & Masks & $\begin{array}{c}\text { Time } \\
(\mathrm{hr})\end{array}$ & $\mathrm{N}_{G C}$ & $\begin{array}{c}<\mathrm{V}> \\
(\mathrm{km} / \mathrm{s})\end{array}$ & $\begin{array}{c}\sigma \\
(\mathrm{km} / \mathrm{s})\end{array}$ \\
\hline 720 & 5 & 10.65 & 65 & 1751 & 168 \\
821 & 7 & 10.17 & 68 & 1743 & 161 \\
1023 & 5 & 8.82 & 113 & 626 & 153 \\
1400 & 4 & 7.61 & 68 & 605 & 131 \\
1407 & 11 & 19.20 & 374 & 1771 & 238 \\
2768 & 6 & 11.50 & 107 & 1328 & 160 \\
2974 & 5 & 8.67 & 26 & 1860 & 128 \\
3115 & 5 & 9.54 & 150 & 710 & 166 \\
3377 & 5 & 11.66 & 126 & 682 & 106 \\
3607 & $5^{a}$ & $10.07^{a}$ & 39 & 976 & 158 \\
& & & & & \\
\hline
\end{tabular}

Table 6 continued 
Table 6 (continued)

\begin{tabular}{rcrrcc}
\hline \hline $\begin{array}{c}\text { Galaxy } \\
(\text { NGC })\end{array}$ & Masks & $\begin{array}{c}\text { Time } \\
(\mathrm{hr})\end{array}$ & $\mathrm{N}_{G C}$ & $\begin{array}{c}<\mathrm{V}> \\
(\mathrm{km} / \mathrm{s})\end{array}$ & $\begin{array}{c}\sigma \\
(\mathrm{km} / \mathrm{s})\end{array}$ \\
\hline 3608 & 5 & 10.07 & 29 & 1195 & 165 \\
4111 & 4 & 8.00 & 15 & 833 & 124 \\
4278 & 6 & 9.92 & 269 & 638 & 191 \\
4365 & 7 & 9.26 & 245 & 1218 & 223 \\
4374 & 3 & 5.50 & 41 & 1171 & 334 \\
4459 & 3 & 6.50 & 36 & 1172 & 137 \\
4473 & 4 & 8.75 & 105 & 2273 & 130 \\
4474 & 3 & 5.70 & 23 & 1613 & 56 \\
4486 & 5 & 13.42 & 653 & 1324 & 380 \\
4494 & 5 & 8.08 & 105 & 1342 & 92 \\
4526 & 4 & 8.00 & 107 & 588 & 175 \\
4564 & 3 & 4.50 & 26 & 1185 & 116 \\
4649 & 4 & 6.67 & 423 & 1097 & 256 \\
4697 & 3 & 5.30 & 90 & 1244 & 151 \\
5846 & 7 & 10.47 & 211 & 1720 & 245 \\
5866 & 1 & 1.00 & 20 & 747 & 127 \\
7457 & 5 & 10.65 & 40 & 839 & 79 \\
& & & & & \\
\hline
\end{tabular}

${ }^{a}$ NGC 3607 globular clusters were obtained from the NGC 3608 masks.

Note-Number of unique DEIMOS masks and total integration time, number of unique confirmed globular clusters, mean heliocentric velocity and the error on the mean, and the velocity dispersion of globular cluster system. Note that the final catalogs of NGC 3115, NGC 4649 and NGC 4486 include data from telescopes/instruments other than DEIMOS.

In Figure 1 we examine the difference between the mean velocity of the GC system with the galaxy systemic velocity as a function of the number of GCs observed. Each galaxy is coded by its Hubble type from Table 1. Most GC systems have a mean velocity that is similar to that of their host galaxy. The main outlier in our sample is NGC 4374 for which we have only $41 \mathrm{GCs}$, and so we suspect that this discrepancy is due to our limited and biased coverage of the GC system. There no obvious trend with Hubble type or number of GCs observed (beyond the expected larger scatter for smaller sample sizes). We conclude that, overall, our GC radial velocity datasets are representative of the GC system dynamics and that they are qualitatively consistent with being in dynamical equilibrium within the galaxy potential. Future work will investigate this issue in more detail and in particular whether substructure (e.g. due to a past merger) is present in these GC systems. For example, in the case of a recent major merger, a 'ringing effect' is expected (A. Burkert 2016, priv. comm.) whereby GCs at large radii will deviate to positive and negative velocities as they settle into equilibrium.

Early-type galaxies are well known to display a relationship between their luminosity (or stellar mass) and the velocity dispersion of their stars. This is commonly called the Faber-Jackson relation (Faber \& Jackson 1976). For typical early-type galaxies the scaling is $\mathrm{M}_{*} \propto \sigma^{4}$, but for the most massive galaxies the scaling steepens to an exponent of $\sim 8$ (Kormendy \& Bender 2013). In Figure 2 we show the relation between the velocity dispersion of the GC system and galaxy stellar mass. Stellar masses are calculated from the total $3.6 \mu \mathrm{m}$ luminosity with an age dependent mass-to-light ratio (Forbes et al. 2016). A Faber-Jackson style $\sigma^{4}$ relation is overplotted, showing that the GC system of typical early-type galaxies obeys a similar relation and that it steepens towards the more massive galaxies. For other kinematic scaling relations between GC systems and their host galaxies see Pota et al. (2013a,b).

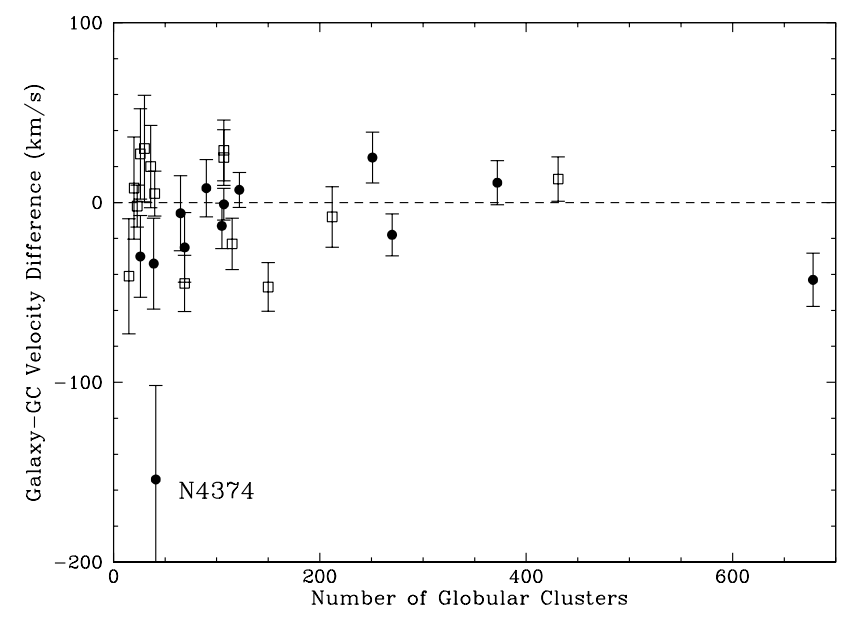

Figure 1. Galaxy systemic velocity minus globular cluster system mean velocity vs number of GCs $(\mathrm{N})$ with radial velocities. Error bars represent globular cluster system velocity dispersion divided by $\sqrt{N}$. Symbols are coded by Hubble type (filled circles for ellipticals, and open squares for S0s and E/S0). The GC systems and their host galaxy have similar mean velocities, with the main outlier being NGC 4374. There is no strong trend with the number of GCs observed or Hubble type.

\section{SUMMARY}

After removing foreground stars, background galaxies and suspected ultra-compact dwarfs from our object lists, we present catalogs of over 4000 globular cluster (GC) radial velocities and positions for the SLUGGS early-type galaxies. Phase space diagrams for each 


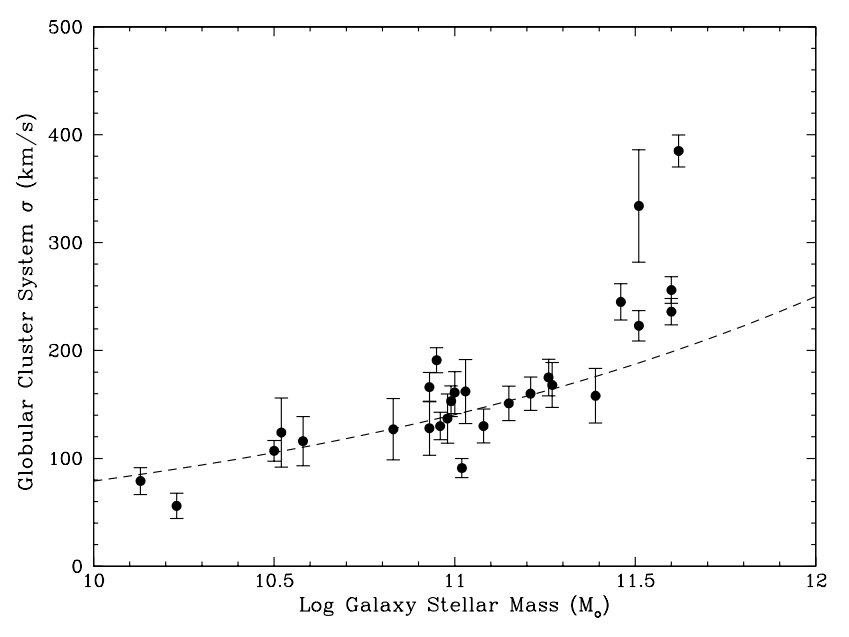

Figure 2. Galaxy stellar mass vs globular cluster system velocity dispersion. The dashed line is not a fit, but it shows a $\mathrm{M}_{*} \propto \sigma^{4}$ relation. The most massive galaxies have a steeper relation. Errors are globular cluster system velocity dispersion divided by $\sqrt{N}$, where $\mathrm{N}$ is the number of GCs. The galaxy with the largest error bar is NGC 4374.

galaxy indicate that contamination from nearby galaxies is low. We show that the mean velocity of the GC system is closely aligned with the systemic velocity of the host galaxy, and that the velocity dispersion of the GC system scales with host galaxy mass similar to the well-known Faber-Jackson relation. We hope that these data prove useful in future studies of GC systems. As new data are obtained we plan to make them available on the SLUGGS website http://sluggs.swin.edu.au

We thank S. Kartha, Z. Jennings, J. Arnold and past members of the SLUGGS survey for their help over the years in acquiring this data. The referee is thanked for a careful reading and several useful suggestions. We thank the staff of the Keck Observatory for their expertise and help over the years collecting these data. The data presented herein were obtained at the W.M. Keck Observatory, which is operated as a scientific partnership among the California Institute of Technology, the University of California and the National Aeronautics and Space Administration. The Observatory was made possible by the generous financial support of the W.M. Keck Foundation. The authors wish to recognize and acknowledge the very significant cultural role and reverence that the summit of Mauna Kea has always had within the indigenous Hawaiian community. We are most fortunate to have the opportunity to conduct observations from this mountain. DAF thanks the ARC for financial support via DP130100388. JPB and AJR acknowledges the NSF grants AST-1211995, AST-1616598 and AST1518294. JS acknowledges the NSF grant AST-1514763 and a Packard Fellowship. CU gratefully acknowledges financial support from the European Research Council (ERC-CoG-646928, Multi-Pop).

Facilities:
Keck(DEIMOS)

\section{REFERENCES}

Alabi, A. B., Foster, C., Forbes, D. A., et al. 2015, MNRAS, 452, 2208

Alabi, A. B., Forbes, D. A., Romanowsky, A. J., et al. 2016, MNRAS, 460, 3838

Arnold J. A., et al., 2014, ApJ, 791, 80

Beasley, M. A., Bridges, T., Peng, E., et al. 2008, MNRAS, 386, 1443

Blom, C., Spitler, L. R., \& Forbes, D. A. 2012, MNRAS, 420,37

Blom C., Forbes D. A., Foster C., Romanowsky A. J., Brodie J. P., 2014, MNRAS, 439, 2420

Bridges, T. J., Rhode, K. L., Zepf, S. E., \& Freeman, K. C. 2007, ApJ, 658, 980

Brodie, J. P., Romanowsky, A. J., Strader, J., et al. 2014, ApJ, 796, 52

Brown, W. R., Geller, M. J., Kenyon, S. J., \& Diaferio, A. 2010, AJ, 139, 59

Cooper, M. C., Newman, J. A., Davis, M., Finkbeiner, D. P., \& Gerke, B. F. 2012, Astrophysics Source Code Library, ascl:1203.003

Côté, P., McLaughlin, D. E., Hanes, D. A., et al. 2001, ApJ, 559, 828

Côté, P., McLaughlin, D. E., Cohen, J. G., \& Blakeslee, J. P. 2003, ApJ, 591, 850

Dowell, J. L., Rhode, K. L., Bridges, T. J., et al. 2014, AJ, 147, 150

Faber, S. M., \& Jackson, R. E. 1976, ApJ, 204, 668

Faber, S. M., Phillips, A. C., Kibrick, R. I., et al. 2003, Proc. SPIE, 4841, 1657

Forbes, D., Pota V., Usher C., Strader J., Romanowsky A. J., Brodie J. P., Arnold J. A., Spitler L. R., 2013, MNRAS, 435, L6

Forbes D. A., Almeida A., Spitler L. R., Pota V., 2014, MNRAS, 442, 1049

Forbes, D. et al. 2016, MNRAS, in press

Foster, C., Spitler, L. R., Romanowsky, A. J., et al. 2011, MNRAS, 415, 3393

Foster C., et al., 2016, MNRAS, 457, 147

Hesser, J. E., Harris, H. C., \& Harris, G. L. H. 1986, ApJL, 303, L51

Huchra, J., \& Brodie, J. 1984, ApJ, 280, 547

Huchra, J., \& Brodie, J. 1987, AJ, 93, 779

Jennings, Z. G., Strader, J., Romanowsky, A. J., et al. 2014, AJ, 148, 32

Kartha, S. S., Forbes, D. A., Spitler, L. R., et al. 2014, MNRAS, 437, 273 
Kartha, S. S., Forbes, D. A., Alabi, A. B., et al. 2016, MNRAS, 458, 105

Kormendy J., Bender R., 2013, ApJ, 769, L5

Misgeld I., Mieske S., Hilker M., Richtler T., Georgiev

I. Y., Schuberth Y., 2011, A\&A, 531, A4

Mould, J. R., Oke, J. B., \& Nemec, J. M. 1987, AJ, 93, 53

Napolitano, N. R., Pota, V., Romanowsky, A. J., et al. 2014, MNRAS, 439, 659

Pastorello, N., Forbes, D. A., Foster, C., et al. 2014, MNRAS, 442, 1003

Pota, V., Forbes, D. A., Romanowsky, A. J., et al. 2013a, MNRAS, 428, 389

Pota V., Graham A. W., Forbes D. A., Romanowsky A. J., Brodie J. P., Strader J., 2013b, MNRAS, 433, 235

Pota, V., Brodie, J. P., Bridges, T., et al. 2015, MNRAS, 450, 1962

Richtler T., Salinas R., Misgeld I., Hilker M., Hau G. K. T., Romanowsky A. J., Schuberth Y., Spolaor M., 2011, A\&A, 531, A119

Richtler T., Hilker M., Kumar B., Bassino L. P., Gómez M., Dirsch B., 2014, A\&A, 569, A41

Schuberth, Y., Richtler, T., Hilker, M., et al. 2010, A\&A, 513, A52

Schuberth Y., Richtler T., Hilker M., Salinas R., Dirsch B., Larsen S. S., 2012, A\&A, 544, A115
Strader, J., Romanowsky, A. J., Brodie, J. P., et al. 2011, ApJS, 197, 33

Tonry, J., \& Davis, M. 1979, AJ, 84, 1511

Usher, C., Forbes, D. A., Brodie, J. P., et al. 2012, MNRAS, 426, 1475

Usher, C., Forbes, D. A., Spitler, L. R., et al. 2013, MNRAS, 436, 1172

Woodley, K. A., Gómez, M., Harris, W. E., Geisler, D., \& Harris, G. L. H. 2010, AJ, 139, 1871

Zepf, S. E., Beasley, M. A., Bridges, T. J., et al. 2000, AJ, 120, 2928

\section{APPENDIX}

In Figures 3-9 we show the distributions of GCs in phase space, i.e. velocity vs projected galactocentric radius for individual host galaxies (see Alabi et al. 2016 for a summary plot stacked by galaxy mass). We also show the galaxy systemic velocity, effective radius and the location of neighbor galaxies (from Table 3). The GC systems generally have a velocity distribution that is symmetric about the galaxy systemic velocity, but there are some notable exceptions e.g. NGC 4374 as highlighted in Figure 1. These plots show that the contribution of GCs from the neighbor galaxies to the overall GC system of the primary SLUGGS galaxy is negligible. 

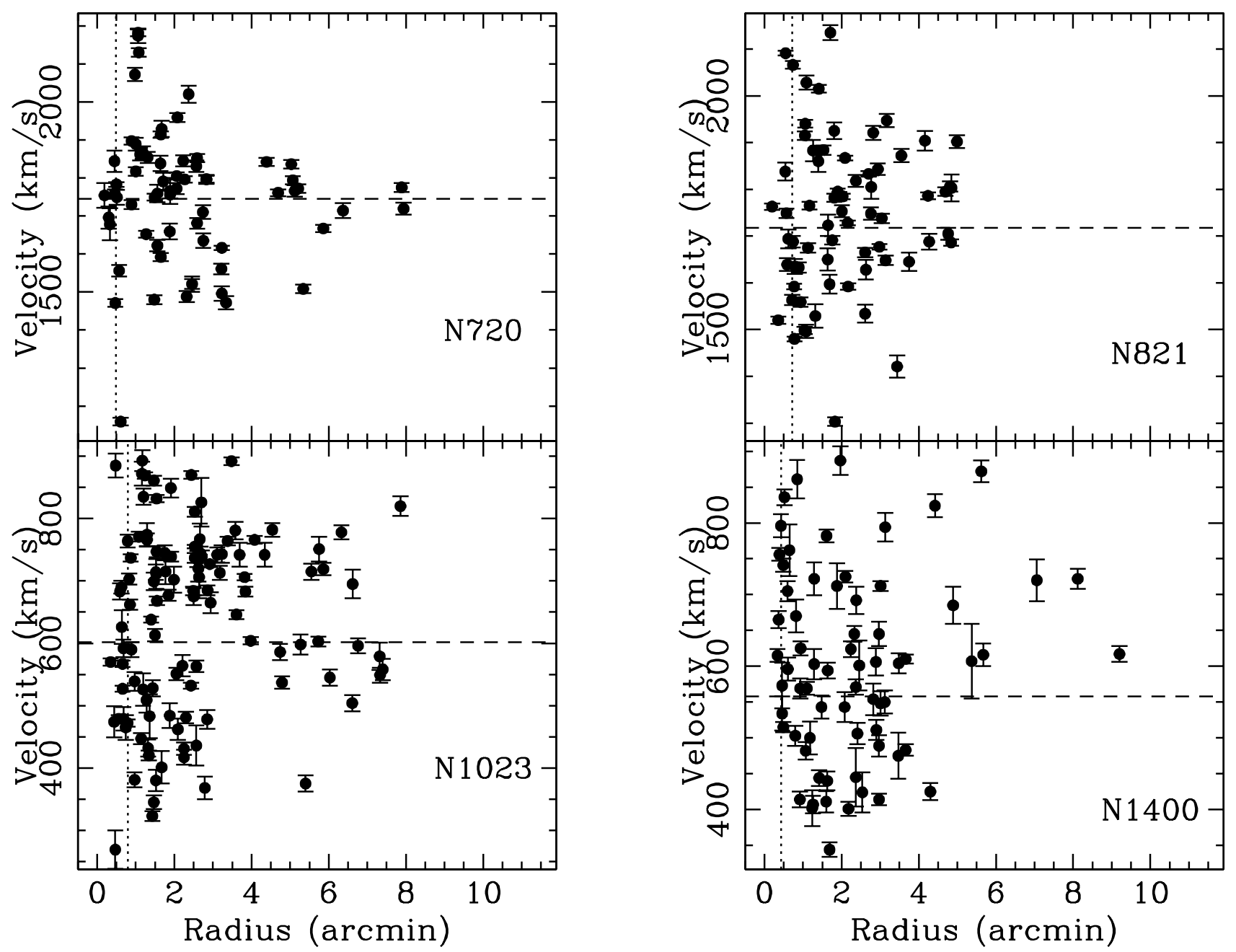

Figure 3. Phase space diagram of GCs associated with NGC 720, NGC 821, NGC 1023 and NGC 1400. Small crosses indicate the location of neighbor galaxies as listed in Table 3. Ultra-compact dwarfs have been omitted from these diagrams. The horizontal dashed line indicates the systemic velocity of the host galaxy, and the vertical dotted line represents the effective radius of the host galaxy. 

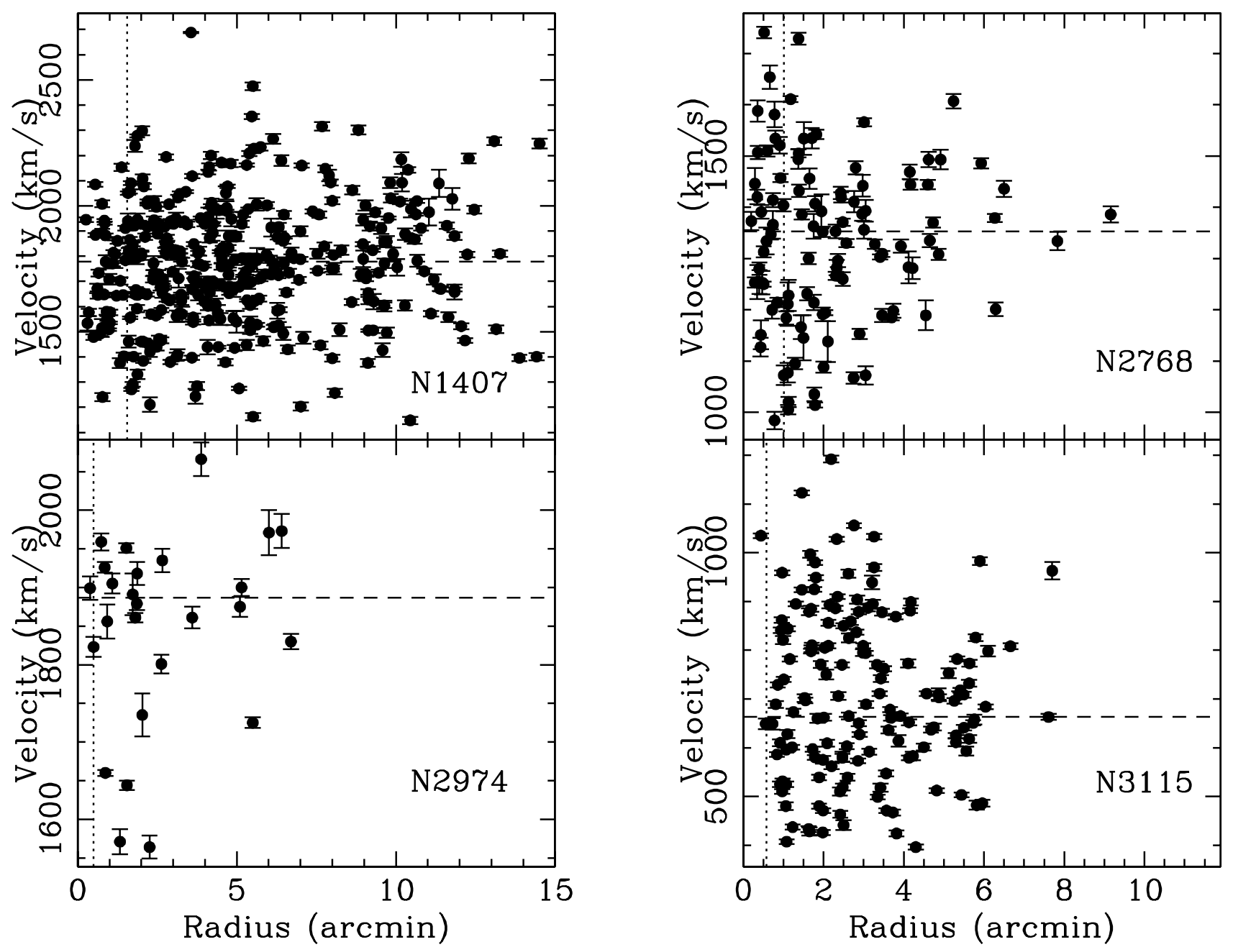

Figure 4. Phase space diagram of GCs associated with NGC 1407, NGC 2768, NGC 2974 and NGC 3115 

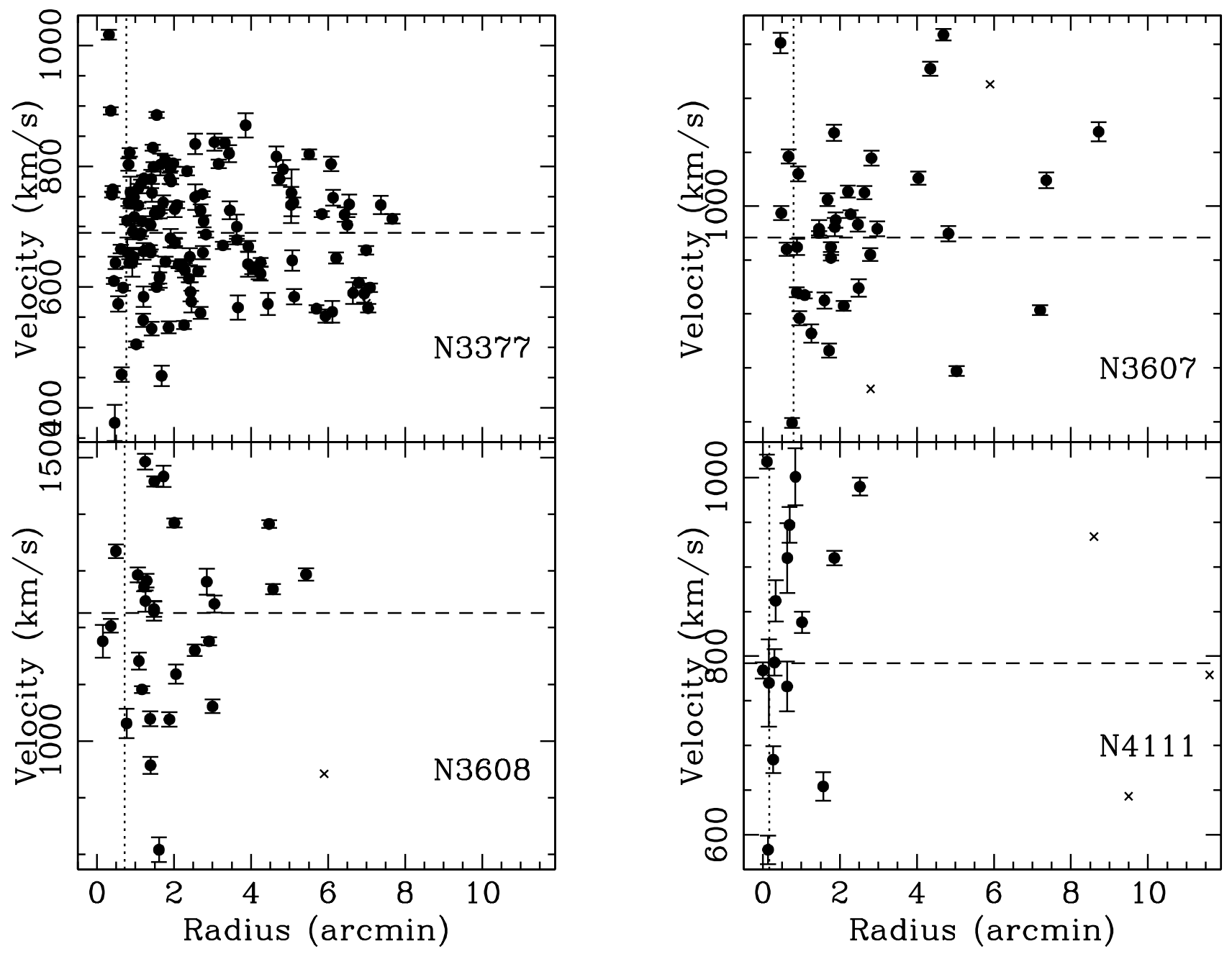

Figure 5. Phase space diagram of GCs associated with NGC 3377, NGC 3607, NGC 3608 and NGC 4111. 

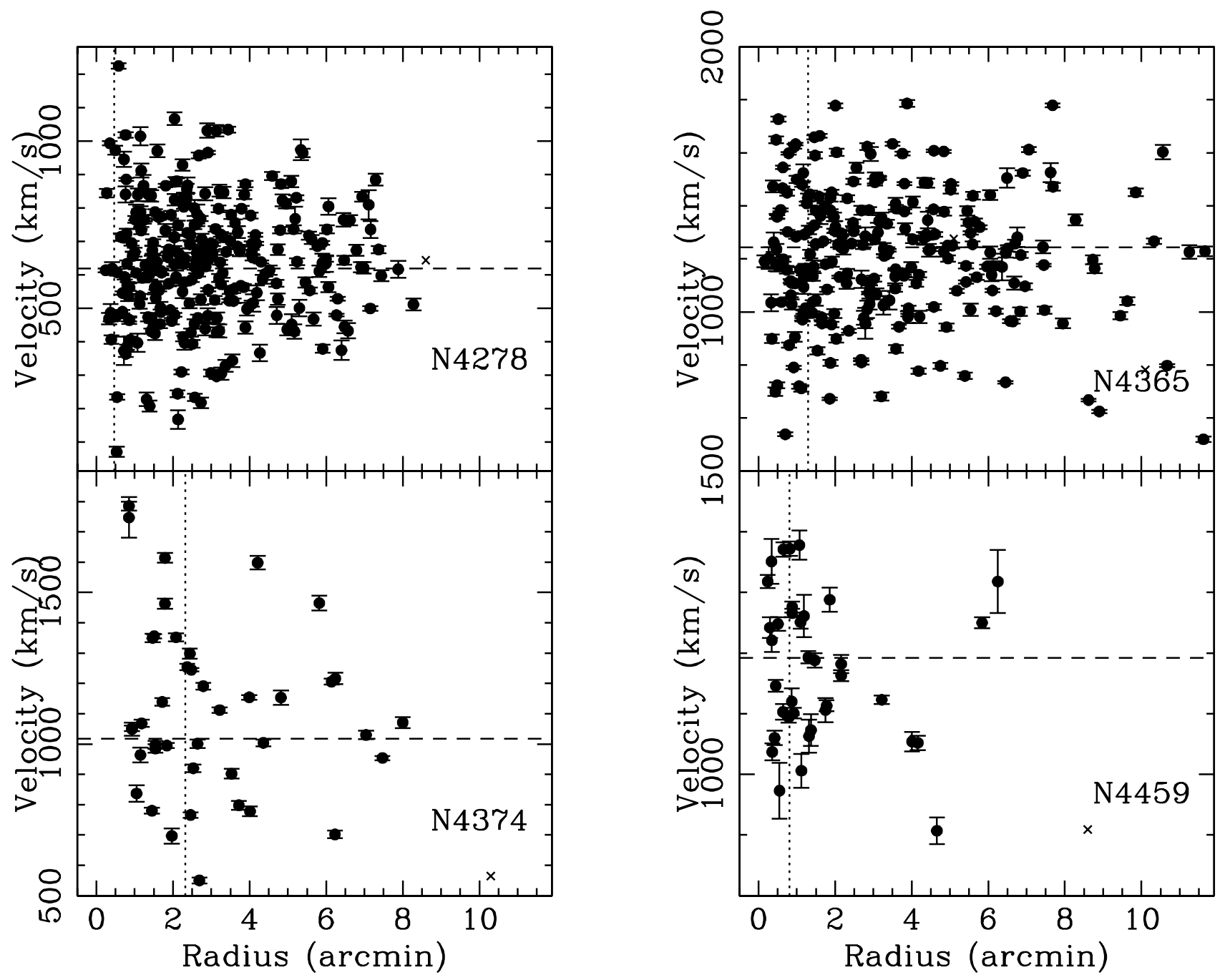

Figure 6. Phase space diagram of GCs associated with NGC 4278, NGC 4365, NGC 4374 and NGC 4459. 

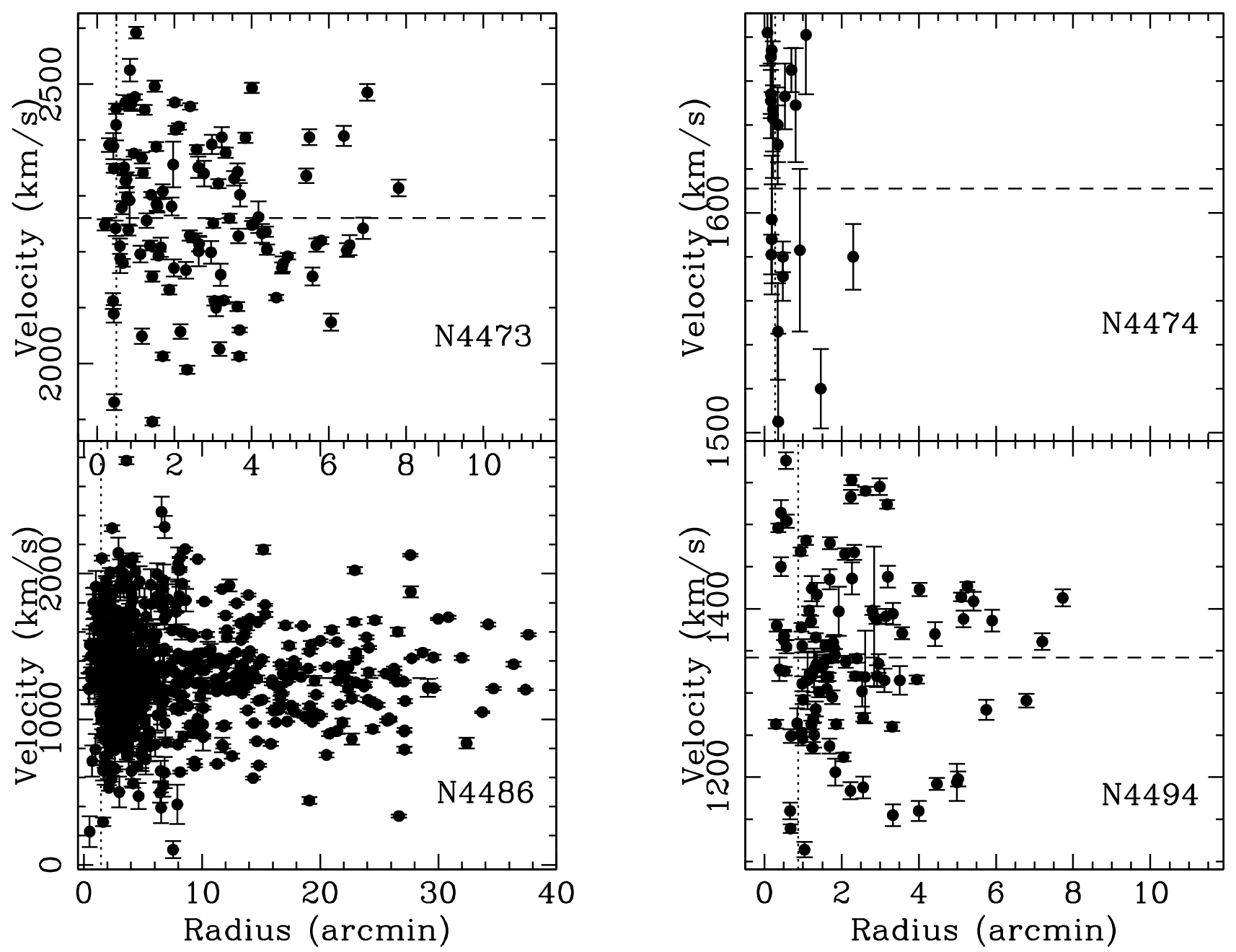

Figure 7. Phase space diagram of GCs associated with NGC 4473, NGC 4474, NGC 4486 and NGC 4494. 

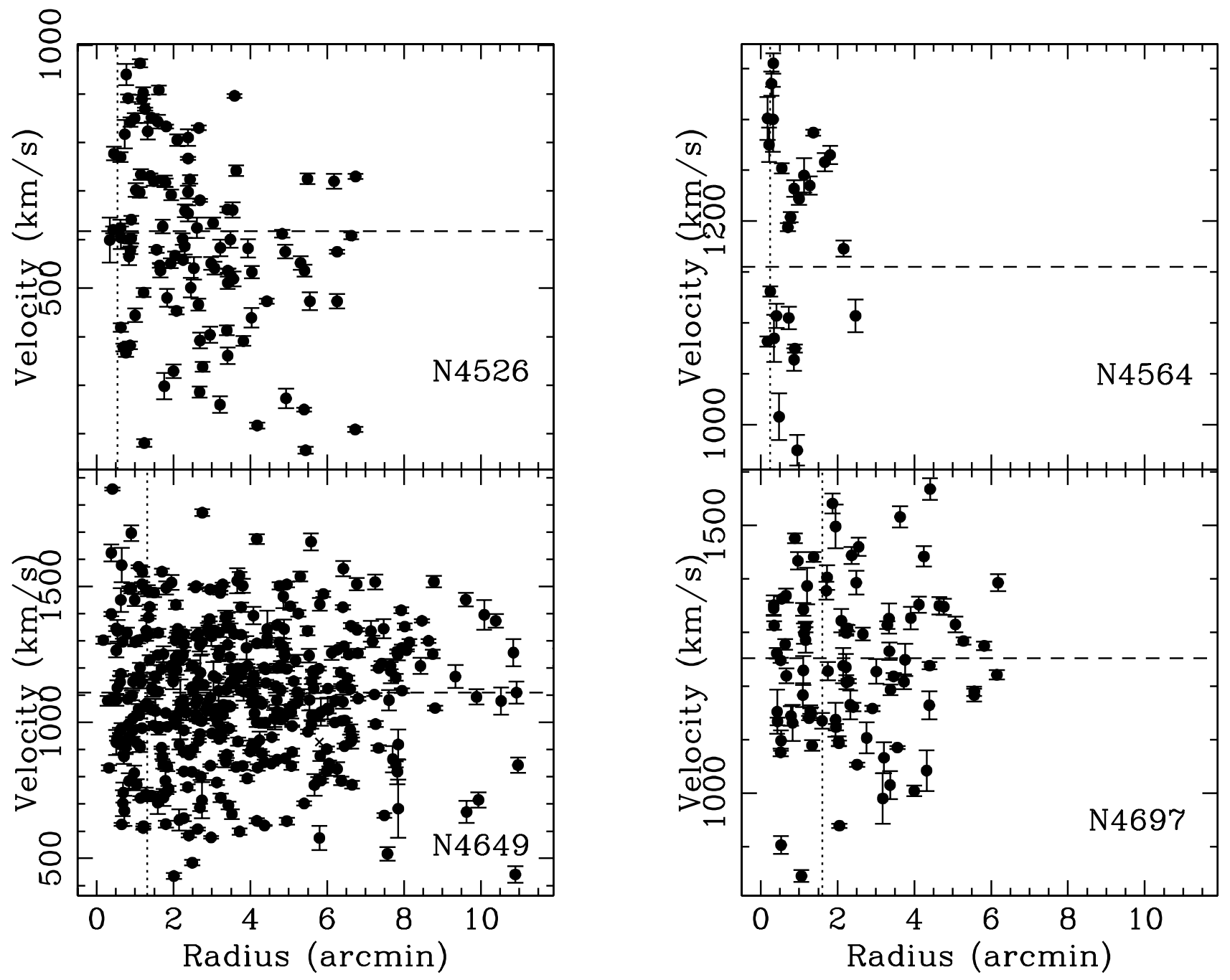

Figure 8. Phase space diagram of GCs associated with NGC 4526, NGC 4564, NGC 4649 and NGC 4697. 

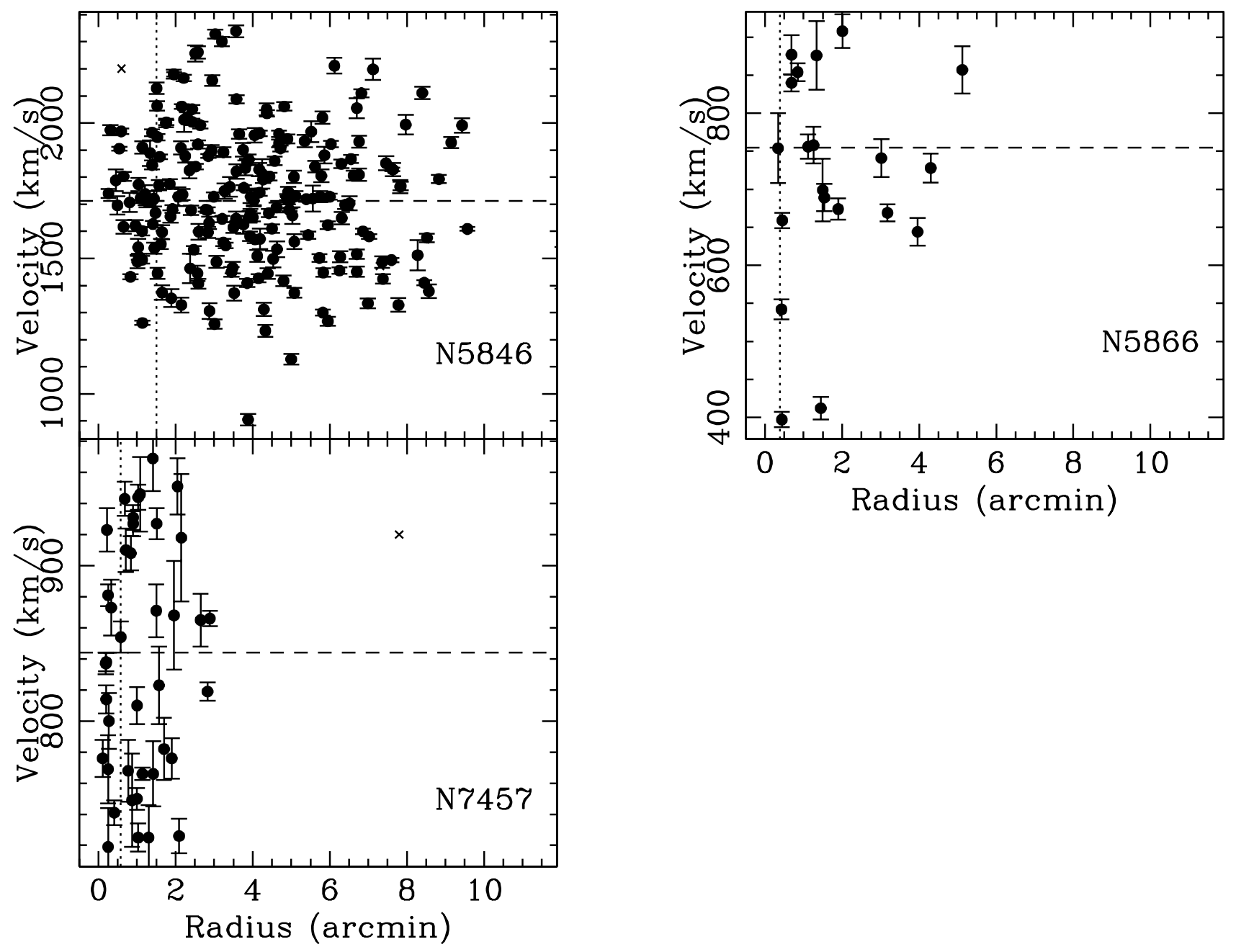

Figure 9. Phase space diagram of GCs associated with NGC 5846, NGC 5866 and NGC 7457. 